\title{
SYSTEMIC CYCLOSPORINE IN HIGH-RISK KERATOPLASTY: LONG-TERM RESULTS
}

\author{
JOHN C. HILL \\ Cape Town, South Africa
}

\begin{abstract}
SUMMARY
Systemic cyclosporine (CSA) was given to 43 high-risk keratoplasty patients (vascularisation in three or four quadrants and >16 stromal vessels): 14 received CSA for 12 months (long CSA group) and 29 for a 4-6 month period (short CSA group). A group of 37 similar high-risk keratoplasty patients received no CSA (no CSA group). In the no CSA group $27(73 \%)$ grafts had rejection episodes compared with $21(48.8 \%)$ in the combined CSA group $(p=0.025)$. Rejection was reversed in only $23.3 \%$ of the no CSA group, compared with $50 \%$ and $87.5 \%$ in the short $(p=0.06)$ and long $(p=0.002)$ CSA groups respectively. Compared with the no CSA group, overall graft survival was better in both the short $(p=0.019)$ and long $(p=0.0056)$ CSA groups. This improvement resulted from both a reduction in the incidence, and an increase in the reversal rate, of rejection episodes. The improvement continued after stopping CSA, suggesting that some immunological privilege had been reestablished. Acuities of 20/40 or better were achieved by $44.8 \%$ and $50 \%$ of eyes in the short and long CSA groups respectively, compared with $13.5 \%$ of eyes in the no CSA group.
\end{abstract}

In keratoplasty the commonest cause of graft failure is rejection. ${ }^{1,2}$ In high-risk keratoplasty the failure rate from rejection is between $60 \%$ and $90 \%$, depending on the criteria for defining high risk..$^{2-6}$ Despite the use of corticosteroids in high doses both topically and systemically, rejection in high-risk keratoplasty is not reliably prevented. Other recent attempts to improve graft prognosis in high-risk cases by tissue matching 7 or by the use of topical cyclosporine (CSA) have not led to a significant improvement.

We have reported on the use of systemic CSA in high-risk keratoplasty ${ }^{8,9}$ and have shown that it improves graft survival by reducing the incidence of

Correspondence to: Dr John C. Hill, Department of Ophthalmology, Medical School, University of Cape Town, Cape Town, South Africa. failure from irreversible graft rejection. ${ }^{9}$ If rejection can be prevented during the early post-operative period, a time when rejection is more likely to occur, then later some degree of immunological privilege may be re-established allowing CSA to be safely withdrawn. Our studies have shown that a 12 month course of systemic CSA is more effective in this regard than the shorter 4-6 month course of therapy. ${ }^{9}$ CSA is a potent immunosuppressant agent with a high degree of specificity for $\mathrm{T}$ lymphocytes. It suppresses cell-mediated reactions and in transplantation it is thought to prevent the early stages of antigenic sensitisation and the resulting proliferation of immunocompetent $\mathrm{T}$ cells. ${ }^{10}$ Therefore, CSA should prevent rejection episodes from occurring. Our studies indicated that only 1 of 43 patients had an irreversible graft rejection while taking CSA, but a larger number of grafts developed reversible rejection episodes. In high-risk keratoplasty it is not known whether systemic CSA reduces the incidence of rejection episodes or whether the incidence remains high but CSA influences the outcome by making the treatment of rejection episodes more successful. This present study investigates these aspects of CSA therapy and examines graft survival to determine whether the beneficial effects of CSA continue after therapy is stopped.

Although rejection remains the commonest cause of graft failure in high-risk keratoplasty, associated ocular damage may lead to graft failure from other causes such as glaucoma, tear film abnormalities or recurrent scarring. If CSA prevents failure from rejection there is the possibility that failure from other causes will become more of a problem. This present study investigates these aspects by analysing graft failure from all causes and documenting the final visual acuity, in a group of high-risk keratoplasty patients given systemic CSA. The results are compared with those in a group of high-risk keratoplasty patients who did not receive CSA. 
Table I. Patient details

\begin{tabular}{|c|c|c|c|c|}
\hline & & Age (years) & Previous grafts & Follow-up ${ }^{\mathrm{a}}$ (months) \\
\hline Group & $n$ & Mean (range) & Mean (range) & Mean (range) \\
\hline No CSA & 37 & $33.6(4-69)$ & $0.57(0-3)$ & $29.6 \quad(6-63)$ \\
\hline Short CSA & 29 & $35.3(4-66)$ & $1.62(0-4)$ & $26.7 \quad(7-49)$ \\
\hline Long CSA & 14 & $24.5(7-42)$ & $1.86(0-5)$ & $52.8(14-82)$ \\
\hline
\end{tabular}

${ }^{a}$ Follow-up refers to clear grafts only.

\section{PATIENTS AND METHODS}

The patients in this prospective study are the same cohort as those reported in a previous study. ${ }^{9}$ All were high-risk keratoplasty patients: defined as having three or four quadrants of superficial and deep corneal vascularisation with the total number of vessels reaching the proposed donor site exceeding 16. Many of them had had previously rejected corneal grafts and all had visual acuities of less than $20 / 200$ in the affected eye. Patients were allocated to a treatment group according to the vision in the other eye. CSA was offered only to patients who were either uniocular or had bilateral corneal disease, with the exception of 3 patients who had a seeing contralateral eye but required binocular vision for their employment. Patients with a good contralateral eye were not offered CSA but received a graft with standard treatment; these grafts were used as a comparison in this study (no CSA group). The number of previous grafts in each group is shown in Table I and the aetiology of the original corneal disease is shown in Table II.

Forty-three patients were given CSA; at the beginning of the study CSA was given for 12 months but because of our initial success it was felt that the length of treatment could be reduced to economise and prevent potential side-effects. Consequently the remaining patients were treated with CSA for 4-6 months. Fourteen patients received CSA for 12 months (long CSA group) and 29 patients received the drug for 4-6 months (short CSA group). In both groups the dose of CSA was administered twice daily by mouth, except for the first dose, which was given intravenously at the time of surgery. An initial loading dose of $15 \mathrm{mg} / \mathrm{kg}$ per day was given for 2 days followed by $7.5 \mathrm{mg} / \mathrm{kg}$ per day for 2 days; thereafter the dose was adjusted to maintain therapeutic blood levels. The method used by our laboratory to estimate CSA blood levels changed a number of times during this study. Initially serum levels were measured, but at present whole blood values are preferred. The current method used is the specific whole blood method ${ }^{11}$ and the therapeutic range of our laboratory is a trough CSA level of $130-250 \mu \mathrm{g} / \mathrm{l}$. We attempt to keep blood levels at the lower end of the therapeutic range, and at present a value of between 130 and $170 \mu \mathrm{g} / \mathrm{l}$ is aimed for; the usual dose to achieve this is approximately $3-4 \mathrm{mg} / \mathrm{kg}$ per day. During the early post-operative period blood CSA levels, together with serum chemistry, were estimated twice weekly. When the blood CSA levels became stabilised, usually at 3 or 4 weeks, the frequency of the tests was reduced. This CSA dosage regimen was maintained for the duration of the treatment period.

A group of 37 patients who had similar high-risk corneas but who had unilateral disease only and were not given CSA, received topical corticosteroids alone (no CSA group).

All the patients in this study were healthy with no serious systemic disease. Those patients receiving CSA underwent a full blood and metabolic investigation, with particular attention being given to the detection of renal and hepatic disease. The keratoplasty techniques were identical for all patients; donor grafts were between 7.5 and $8.5 \mathrm{~mm}$ and were $0.5 \mathrm{~mm}$ larger than the recipient bed. Donor corneas were preserved as corneoscleral discs in culture medium (M.K. or KSol solution) at $4{ }^{\circ} \mathrm{C}$. A number of patients had a simultaneous extracapsular cataract extraction with the placement of a posterior chamber intraocular lens. All surgery was performed by the author and tissue matching of donor and recipient was not undertaken. A viscoelastic substance was used to protect donor endothelium and to dissect any iris adhesions. A 10/0 nylon suture was used to secure the graft using interrupted or continuous sutures or a combination of both. The anterior chamber was reformed with balanced salt solution, and subconjunctival injections of gentamicin $20 \mathrm{mg}$ and betamethasone (Celestone) $2 \mathrm{mg}$ were given at completion of surgery.

All patients were given topical dexamethasone $0.1 \%$ drops 4 times daily and betamethasone $0.1 \%$ ointment at night. The drops were reduced slowly over the ensuing months and stopped at 6 months unless persistent inflammation required extended therapy. The ointment was usually stopped after 2 weeks.

Table II. Original aetiology

\begin{tabular}{lccc}
\hline & Long CSA & Short CSA & No CSA \\
\hline Herpetic keratitis & 1 & 2 & 3 \\
Chemical burn & 4 & 6 & 8 \\
Keratoconus & 4 & 3 & 2 \\
Bullous keratopathy & 0 & 3 & 3 \\
Trachoma & 0 & 2 & 3 \\
Interstitial keratitis & 0 & 4 & 10 \\
Scarring post keratitis & 5 & 7 & 5 \\
Other & 0 & 2 & 3 \\
\hline
\end{tabular}


Table III. Number of grafts with rejection episodes

\begin{tabular}{|c|c|c|c|c|}
\hline \multirow[b]{2}{*}{ Group } & \multirow[b]{2}{*}{$n$} & Grafts rejecting & \multicolumn{2}{|c|}{ Time to rejection ${ }^{\mathrm{a}}$ (months) } \\
\hline & & No. $(\%)$ & Mean & Median \\
\hline No CSA & 37 & $27 \quad(73.0 \%)$ & 9.1 & 6 \\
\hline Short CSA & 29 & $(51.7 \%)$ & 11.3 & 8 \\
\hline Long CSA & 14 & $(42.6 \%)$ & 12.0 & 10 \\
\hline Total CSA & 43 & $(48.8 \%)^{\mathrm{b}}$ & 11.5 & 8 \\
\hline
\end{tabular}

${ }^{\mathrm{a}}$ Time until the first rejection episode if more than one.

${ }^{\mathrm{b}}$ This result is significant $(p=0.025)$ compared with the no CSA group.

A rejection episode was diagnosed when an eye with a previously clear, thin graft became inflamed with: flare and cells in the anterior chamber, keratic precipitates limited to the donor endothelium, and thickening of the graft either diffusely (probable rejection ${ }^{16}$ ) or in the form of an advancing rejection line (definite rejection ${ }^{16}$ ). Stromal infiltration without epithelial disease was also considered to be a probable rejection episode. The treatment of rejection consisted of hourly dexamethasone $0.1 \%$ drops and a subconjunctival injection of $2 \mathrm{mg}$ betamethasone (Celestone). Eyes with a history of herpes simplex keratitis were also treated with topical antiviral medication. In severe cases of endothelial rejection oral prednisone $60 \mathrm{mg}$ daily was given in addition to the topical medication. Treatment was continued until the inflammation subsided and was then gradually withdrawn. Later in the series a $500 \mathrm{mg}$ intravenous pulse of methylprednisolone was substituted for the oral corticosteroids. A graft was deemed irreversibly rejected when it remained thickened and oedematous with severe visual loss despite maximal medical therapy.

In the first part of this study the incidence of graft rejection was studied regardless of whether the reaction was subsequently reversed. Statistical analysis was performed using the log-rank method with the end-point being the commencement of any graft rejection episode. The number of rejection episodes

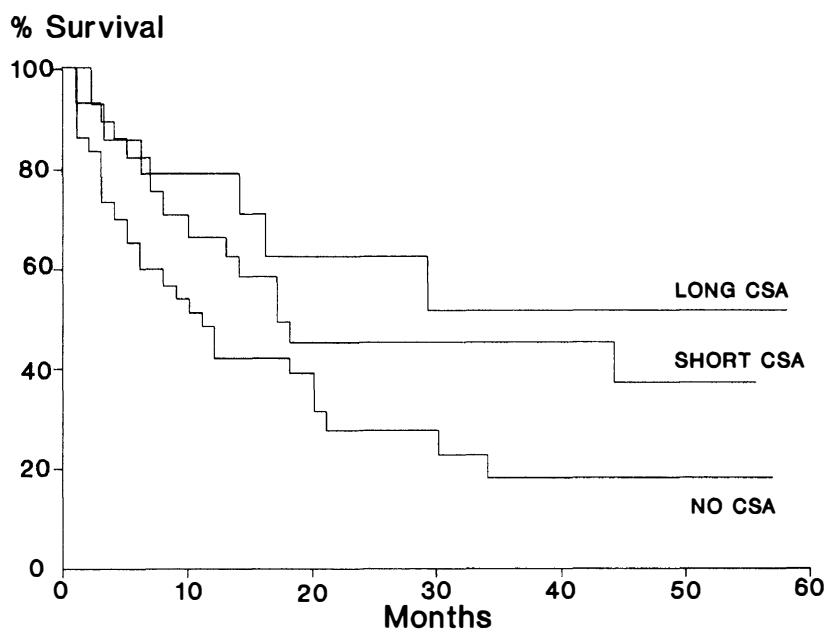

Fig. 1. Survival estimates for the three treatment groups. The end-point is the commencement of a rejection episode. that were reversed by treatment in the three groups was analysed using chi-squared or Fisher's tests.

In the second part of the study graft failure from any cause was studied. The end-point in the survival analysis was the commencement of a rejection episode in a graft that became irreversibly rejected or failure from any other cause. Failure from another cause was defined as a graft complication causing a loss of vision to an acuity of $20 / 60$ or less; the endpoint in the statistical analysis was the time when vision dropped to this level. Graft survival was compared by log-rank analysis and the Cox proportional hazards model.

\section{RESULTS}

The number of grafts that had one or more rejection episodes is shown in Table III. In the no CSA group $27(73 \%)$ of the 37 grafts had a rejection episode, compared with $21(48.8 \%)$ of the 43 grafts in the combined CSA group; the difference is significant $(p=0.025)$. When the total CSA group is subdivided into short and long CSA groups the number of grafts that started to reject was $15(51.7 \%)$ and $6(42.6 \%)$ respectively. There is no statistical difference in survival between the two CSA groups. When survival is compared with the no CSA group, neither the short CSA group ( $p=0.099)$ nor the long CSA group $(p=0.055)$ reach a level of statistical significance. Both the mean and median time to the first rejection episode were shorter in the no CSA group. Fig. 1 shows actuarial survival curves of the three treatment groups; the end-point of survival in these graphs is the commencement of the first rejection episode. Rejection-free survival was better for grafts in the long CSA group compared with grafts in the short CSA group; both CSA groups showed improved survival compared with the no CSA group.

The total numbers of rejection episodes in the three groups are shown in Table IV (note that some

Table IV. Total number of rejection episodes

\begin{tabular}{lcccl}
\hline Group & $n$ & $\begin{array}{c}\text { No. of } \\
\text { episodes }\end{array}$ & Mean & No. reversed (\%) \\
\hline No CSA & 37 & 30 & 0.81 & $7(23.3 \%)$ \\
Short CSA & 29 & 18 & 0.62 & $9(50 \%) \quad p=0.06$ \\
Long CSA & 14 & 8 & 0.57 & $7(87.5 \%) p=0.002$ \\
Total CSA & 43 & 26 & 0.60 & $16(61.5 \%) p=0.009$ \\
\hline
\end{tabular}

$p$ values indicate significance of number reversed compared with the no CSA group. 
Table V. Treatment regimes and outcomes of rejection episodes

\begin{tabular}{lccc}
\hline & \multicolumn{2}{c}{ No. of rejection episodes } & \\
\cline { 2 - 3 } Group regimen $^{\mathrm{a}}$ & Total & Reversed & Rejected \\
\hline No CSA & 7 & 0 & $7(100 \%)$ \\
$\quad$ Topical & 12 & $2(17 \%)$ & $10(83 \%)$ \\
Oral & 11 & $5(45 \%)$ & $6(55 \%)$ \\
$\quad$ Pulse & 1 & 0 & $1(100 \%)$ \\
Short CSA & 5 & $2(40 \%)$ & $3(60 \%)$ \\
$\quad$ Topical & 12 & $7(58 \%)$ & $5(42 \%)$ \\
$\quad$ Oral & 1 & 0 & $1(100 \%)$ \\
Pulse & 2 & $2(100 \%)$ & 0 \\
Long CSA & 5 & $5(100 \%)$ & 0 \\
$\quad$ Topical & & & \\
Oral & & & \\
Pulse & &
\end{tabular}

Topical, hourly topical corticosteroid drops + subconjunctival corticosteroid injection; oral, hourly topical corticosteroid drops + oral corticosteroids; pulse, hourly topical corticosteroid drops + single IV injection of $500 \mathrm{mg}$ methylprednisolone.

asee text for details.

patients had more than one rejection episode). The treatments given for the rejection episodes are shown in Table V. In the no CSA group a total of 30 episodes occurred, giving a mean number of 0.81 per graft; of these 30 episodes only $7(23.3 \%)$ were reversed. In the short CSA group, 18 episodes occurred giving a mean value of 0.62 per graft; 9 $(50 \%)$ of these episodes were reversed with treatment. In the long CSA group there were 8 rejection episodes, giving a mean of 0.57 per graft; 7 (87.5\%) of these episodes were reversed. In the total CSA group there was a statistically better chance of the rejection episode being reversed by treatment, compared with the no CSA group $(p=0.009)$. Taking the two CSA groups separately, the improved rejection reversal rate was also seen in the long CSA group $(p=0.002)$ but the improvement failed to achieve statistical significance in the short CSA group $(p=0.06)$, when compared with the no CSA group. To assess whether this improved rejection reversal rate is maintained after the CSA therapy is stopped, the two CSA groups were analysed before and after cessation of treatment (Table VI). It can be seen that the reversal rates while on CSA are high: $75 \%$ and $100 \%$ for the short and long CSA groups respectively. After treatment is

Table VI. Number of rejection episodes during and after CSA treatment

\begin{tabular}{|c|c|c|}
\hline Group & No. of episodes & No. reversed (\%) \\
\hline No CSA & 30 & $7(23.3 \%)$ \\
\hline \multicolumn{3}{|l|}{ Short CSA } \\
\hline On CSA & 4 & $3 \quad(75 \%)$ \\
\hline Off CSA & 14 & $6 \quad(42.9 \%) p=0.16$ \\
\hline \multicolumn{3}{|l|}{ Long CSA } \\
\hline On CSA & 3 & $3(100 \%)$ \\
\hline Off CSA & 5 & $4(80 \%) \quad p=0.03$ \\
\hline \multicolumn{3}{|l|}{ Total CSA } \\
\hline On CSA & 7 & $6(85.7 \%)$ \\
\hline Off CSA & 19 & $10 \quad(52.6 \%) p=0.07$ \\
\hline
\end{tabular}

$p$ values indicate significance of number reversed compared with the no CSA group.

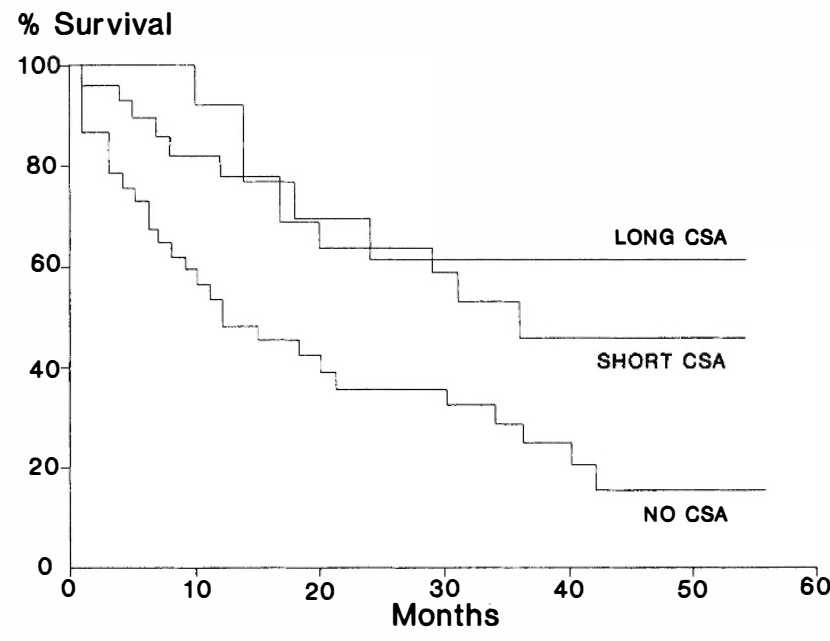

Fig. 2. Survival estimates for the three treatment groups. The end-point is graft failure from any cause.

stopped the reversal rates drop to $42.9 \%$ and $80 \%$ for the short and long CSA groups respectively, but both are better than the rate in the no CSA group $(23.3 \%)$. However, only the results of the long CSA group achieve statistical significance $(p=0.03)$ compared with the no CSA group.

Although irreversible rejection was the commonest cause of graft failure, a number of grafts failed from other causes (Table VII), failure being defined as a visual acuity of $20 / 60$ or worse. The number of grafts that failed for reasons other than rejection are as follows: no CSA group $5(13.5 \%)$, short CSA group $3(8.1 \%)$ and long CSA group 4 (28.6\%). Fig. 2 shows survival curves of the three groups, the endpoint being irreversible graft failure from rejection or failure from any other cause. There was a significantly improved survival of grafts in the short CSA group ( $p=0.019)$, long CSA group $(p=0.0056)$ and combined CSA group $(p=0.0036)$ when compared with the no CSA group. There was no difference between the two CSA groups in terms of survival $(p=0.43)$. Using the Cox proportional hazards

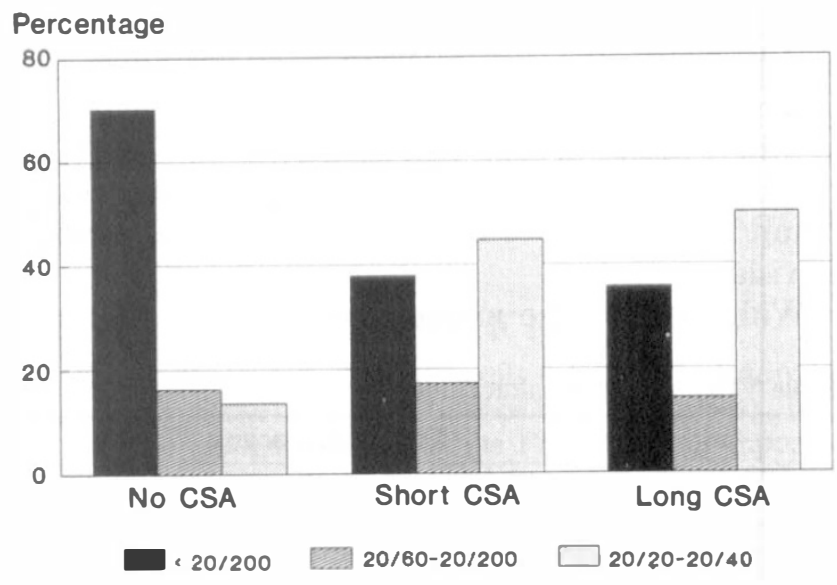

Fig. 3. Final visual acuities in each group, shown as a percentage. 
Table VII. Graft outcome

\begin{tabular}{|c|c|c|c|c|c|}
\hline \multirow[b]{2}{*}{ Group } & \multirow[b]{2}{*}{ No. clear } & \multirow[b]{2}{*}{ No. rejected } & \multicolumn{2}{|c|}{ Other failures } & \multirow{2}{*}{$\frac{\text { Follow-up months }}{\text { Mean (range) }}$} \\
\hline & & & No. & Cause & \\
\hline No CSA & 9 & 23 & 5 & $\begin{array}{l}\text { HSV } \\
\text { HSV } \\
\text { Glaucoma } \\
\text { Bact. scar } \\
\text { Epith. defect }\end{array}$ & $29.6 \quad(6-63)$ \\
\hline Short CSA & 17 & 9 & 3 & $\begin{array}{l}\text { HSV } \\
\text { Bact. scar } \\
\text { Epith. defect }\end{array}$ & $26.7 \quad(7-49)$ \\
\hline Long CSA & 9 & 1 & 4 & $\begin{array}{l}\text { Bact. scar } \\
\text { Epith. defect } \\
\text { Epith. defect } \\
\text { Late decomp. }\end{array}$ & $52.8(14-82)$ \\
\hline
\end{tabular}

HSV, recurrent herpetic keratitis leading to scarring; Bact. scar, bacterial keratitis leading to scarring; Epith. defect, persistent epithelial defect leading to scarring and/or vascularisation in patients with previous chemical burns; Glaucoma, loss of vision from graft damage resulting from uncontrolled IOP; Late decomp., late graft decompensation without inflammation.

model a difference in survival between the CSA groups exists: at any point in time the hazard of a graft in the short CSA group becoming a failure is only $50.2 \%$ that of a graft in the no CSA group; in the long CSA group the hazard is only $25.2 \%$ compared with the no CSA group.

The final visual acuities of the eyes in the three groups is shown in Table VIII and diagrammatically in Fig. 3. Acuities of 20/40 or better were achieved by $44.8 \%$ and $50 \%$ of eyes in the short and long CSA groups respectively. This compared with only $13.5 \%$ of eyes in the no CSA group. The number of eyes achieving $20 / 60$ to $20 / 200$ acuity was similar in the three groups. However, $70.3 \%$ of eyes in the no CSA group had acuities of less than 20/200, compared with $37.9 \%$ and $35.7 \%$ in the short and long CSA groups respectively.

\section{DISCUSSION}

The definition of high-risk in this study, namely three or four quadrants involved with at least 16 vessels passing over the proposed graft site, is stricter than other studies $^{7}$ that define high-risk as two or more quadrants of vascularisation and/or a previously rejected graft in the affected eye. The failure rate in the no CSA group from rejection alone was $62.2 \%$ and from all causes was $75.7 \%$; the final figure is comparable to failure rates of between $60 \%$ and $90 \%$ in other studies of high-risk keratoplasty. ${ }^{2-6}$ In highrisk patients, blind from corneal disease, these high failure rates do not offer much hope for the prospect of vision.

Without CSA the major cause of graft failure in

Table VIII. Final visual acuities

\begin{tabular}{|c|c|c|c|}
\hline \multirow[b]{3}{*}{ Group } & \multicolumn{3}{|c|}{ Final visual acuity } \\
\hline & $<20 / 200$ & $20 / 60-20 / 200$ & $20 / 20-20 / 40$ \\
\hline & $n(\%)$ & $n(\%)$ & $n(\%)$ \\
\hline$\overline{\text { No CSA }}$ & $26(70.3 \%)$ & $6(16.2 \%)$ & $5(13.5 \%)$ \\
\hline Short CSA & $11(37.9 \%)$ & $5(17.2 \%)$ & $13(44.8 \%)$ \\
\hline Long CSA & $5(37.5 \%)$ & $2(14.3 \%)$ & $7(50 \%)$ \\
\hline
\end{tabular}

high-risk keratoplasty remains rejection. In a previous paper $^{9}$ it was shown that systemic CSA effectively prevents graft failure from irreversible graft rejection, especially if treatment is given for 12 months. This present study shows that CSA achieves this result by two actions. Firstly, CSA reduces the number of rejection episodes, as shown by the improved survival of CSA-treated grafts when the commencement of a rejection episode was used as an end-point. Secondly, in CSA-treated grafts the rejection episodes were more effectively reversed compared with the no CSA group. In both respects the outcome of grafts treated with CSA for 12 months was better. It could be expected that while on CSA treatment any rejection episode is more easily reversed because the patient is taking two immunosuppressant drugs (CSA and corticosteroids) whereas patients in the no CSA group are only being given one (corticosteroids). However, even after CSA was stopped the rejection reversal rates remain higher in the CSA-treated patients; possibly because of small numbers, statistical significance was demonstrated only in the long CSA group. The improved survival of grafts, which is maintained after cessation of CSA treatment, indicates that some degree of immunological privilege may have been reestablished.

Early in the study, rejection episodes that did not appear to be severe were treated with topical corticosteroid drops and a subconjunctival steroid injection. These episodes invariably progressed and, despite the addition of oral corticosteroids at a later stage, the grafts all failed from the rejection episode (Table V). The regimen was then changed and all patients with rejection episodes were given systemic steroids when they presented. Initially this was in the form of oral prednisone $60 \mathrm{mg}$ daily but later in the study this was replaced by a single intravenous injection of $500 \mathrm{mg}$ methylprednisolone. Because patients in the no CSA group tended to reject earlier, more of these patients were treated by topical 
steroids alone, and conversely more of the CSA groups were treated with intravenous methylprednisolone. The three treatment regimens used for rejection may have influenced the results because the grafts treated with intravenous methylprednisolone appeared to have fared better - a finding reported by us previously. ${ }^{17}$ All rejecting grafts treated only by local corticosteroids failed, regardless of whether they were treated with CSA. Rejecting grafts in the long CSA group treated with oral steroids or with intravenous methylprednisolone appeared to be more effectively reversed than those in the short CSA group, which in turn fared better than rejecting grafts in the no CSA group. This finding is in agreement with the statement made earlier that in CSA-treated grafts the rejection episodes were more effectively reversed, especially in grafts treated with CSA for 12 months.

Other non-immunological causes of graft failure such as recurrent herpetic keratitis and persistent epithelial defects in dry eyes are important and need to be considered. In the long CSA group, in which irreversible rejection was more effectively prevented, the failure rate from non-immunological causes was higher. It would seem, therefore, that by preventing rejection other diseases may be given the opportunity to become causes of graft failure. Despite this, the survival of CSA-treated grafts, and especially those in the long CSA group, remained statistically better than the no CSA group, taking failure from any cause as the end-point. This improved survival was reflected by the improved final visual acuity results found in the two CSA groups, both of which were substantially better than those of the no CSA group. It should be noted that the follow-up of the long CSA group was longer than for the other two groups, theoretically giving more time for late complications to arise.

The trial was not masked and therefore a patient's treatment group was known to the observers. However, it is unlikely that bias in assessing graft clarity was introduced as the end-points were clearly evident. Grafts that irreversibly rejected invariably developed severe rejection episodes resulting in significant endothelial loss and final corneal thickness measurements in excess of $700 \mu \mathrm{m}$ centrally. In the patients with non-immunological failure the endpoint was clearly defined as a best visual acuity of $20 / 60$ or worse. The diagnosis was made clinically and in those grafts that had associated ocular inflammation no other evidence of rejection, such as a rejection line, keratic precipitates or graft oedema from endothelial loss was present.

CSA is a potent immunosuppressant drug and careful pre-operative systemic investigations and post-operative monitoring are essential to minimise the potential dangers which can arise from its use.
Nephrotoxicity, ${ }^{12,13}$ hepatotoxicity ${ }^{14}$ and hyperten$\operatorname{sion}^{15}$ are known complications of CSA use. In addition the suppression of $\mathrm{T}$ cell function may lead to infections or to re-activation of an existing infection. The complications from the use of CSA in this cohort of patients were few and have been discussed previously. ${ }^{9}$ One patient in the short CSA group developed a TB abscess in the neck, presumably from a re-activation of an undiagnosed inactive lesion; specific TB therapy was commenced and cyclosporine stopped at 2 months. Another patient in the same group developed an intractable pruritic rash and treatment was stopped at 1 month. The grafts from both patients irreversibly rejected: the results were uncensored and are included as failures from rejection while on CSA. Other sideeffects were less severe and did not require cessation of treatment. One patient who was mildly hypertensive required additional therapy and 4 patients had an increase in their serum creatinine levels to outside the normal range, but all returned to normal when the dosage of CSA was reduced. Two female patients complained of hirsutism, which in 1 patient became apparent at 4 months and in the other at 7 months; in both the condition reverted to normal on cessation of treatment. Apart from 1 case of hirsutism all the complications occurred within the first few months of treatment. The longer period of treatment in the long CSA group did not give rise to additional sideeffects, and after 6 months monitoring was reduced to monthly intervals. The low complication rate in this study was possibly due to the good general health of the patients and also because the dosage of CSA used was kept to a minimum by maintaining the blood CSA levels at the lower end of the therapeutic range. The risks of using systemic CSA are therefore justified in high-risk keratoplasty patients who are blind from their disease, are healthy, and who are also willing to comply with treatment and monitoring regimens.

This work was partially funded by a grant from the South African Medical Research Council. The statistical analysis was performed by Dr Sedick Isaacs, PhD, Department of Informatics, Groote Schuur Hospital, Cape Town and Dr Carl Lombard, PhD, MRC Institute of Biostatistics, Cape Town.

Key words: Corneal graft, Cyclosporine, Keratoplasty, Rejection.

\section{REFERENCES}

1. Khodadoust AA. The allograft rejection reaction: the leading cause of late failure of clinical corneal grafts. In: Corneal graft failure. Ciba Foundation Symposium 15, new series. Amsterdam: Elsevier, 1973:151-67.

2. Arentsen JJ. Corneal transplant allograft reaction: possible predisposing factors. Trans Am Ophthalmol Soc 1983;81:361-402.

3. Fine M, Stein M. The role of corneal vascularisation in human corneal graft reactions. In: Corneal graft failure. 
Ciba Foundation Symposium 15, new series. Amsterdam: Elsevier, 1973:193-204.

4. Brown SI, Bloomfield SE, Pearce DB. A follow-up report on transplantation of the alkali-burned cornea. Am J Ophthalmol 1974;77:538-44.

5. Batchelor JR, Casey TA, Gibbs DC, Werb A, Lloyd DF, Prasad SS, James A. HLA matching and corneal grafting. Lancet 1976;1:551-4.

6. Foulks GN, Sanfilippo F. Beneficial effects of histocompatibility in high-risk corneal transplantation. Am J Ophthalmol 1982;94:622-9.

7. The Collaborative Corneal Transplantation Studies Research Group. The Collaborative Corneal Transplantation Studies (CCTS): effectiveness of histocompatibility matching in high-risk corneal transplantation. Arch Ophthalmol 1992;110:1392-403.

8. Hill JC. The use of cyclosporine in high-risk keratoplasty. Am J Ophthalmol 1989;107:506-10.

9. Hill JC. Systemic cyclosporine in high-risk keratoplasty: short versus long term therapy. Ophthalmology 1994;101:128-33.

10. Hess AD, Esa AH, Colombani PM. Mechanism of action of cyclosporine: effect on cells of the immune system and on subcellular events in $\mathrm{T}$ cell activation. Transplant Proc 1988;20(Suppl 2):29-40.

11. Yatscoff RW, Copeland KR, Faraci CJ. Abbott Tdx monoclonal antibody assay evaluated for measuring cyclosporine in whole blood. Clin Chem 1990;36:1969-73.

12. Palestine AG, Austin HA III, Balow JE, Antonovych TT, Sabnis SG, Preuss HG, Nussenblatt RB. Renal histopathologic alterations in patients treated with cyclosporine for uveitis. N Engl J Med 1986; 314:1293-8.

13. Mihatsch MJ, Thiel G, Ryffel B. Histopathology of cyclosporine nephrotoxicity. Transplant Proc 1988;20(Suppl 3):759-71.

14. Vine W, Billiar T, Simmons R, Bowers LD. Cyclosporine-induced hepatotoxicity: a microassay by hepatocytes in tissue culture. Transplant Proc 1988;20(Suppl 3):859-62.

15. Munoz SJ, Vlasses PH, Boullata JI, Zaragoza MR, Jarrell BE, Maddrey WC. Elevated arterial blood pressure in survivors of liver transplantation treated with cyclosporine and corticosteroids. Transplant Proc 1988;20(Suppl 3):623-7.

16. Stulting RD, Waring GO, Bridges WZ, Cavanagh HD. Effect of donor epithelium on corneal transplant survival. Ophthalmology 1988;95:803-12.

17. Hill JC, Maske R, Watson P. Corticosteroids in corneal graft rejection: oral versus single pulse therapy. Ophthalmology 1991;98:329-33. 